\title{
What is volition?
}

\author{
Patrick Haggard · Hakwan Lau
}

Published online: 1 June 2013

(C) Springer-Verlag Berlin Heidelberg 2013

Volition refers to the capacity of humans, and other animals, to initiate actions based on internal decision and motivation, rather than external stimulation. It seems unimaginable that a special issue on volition could have occurred in this journal, or another journal of its field, 20 years ago. The long behaviourist period that dominated thinking about brain and behaviour in the mid-twentieth century had no place for internal states and processes, and volition is an internal process by definition. The cognitivist period that succeeded was also interestingly silent on the topic of volition. Yet voluntary action lies at the heart of most accounts of human nature. Societies assume that individuals are autonomous agents, choosing and initiating their own courses of action, and thus becoming responsible for what they do. Neuroscience remained silent, or even sceptical, about this key feature of human biology and human experience.

\section{Scientific breakthroughs enabling the study of volition}

What, then, has produced the recent explosion of scientific interest in volition? In our view, two scientific developments have played key roles. The first is the importance of measuring volition. Here, the crucial step was taken by Benjamin Libet, who adapted the mental chronometry methods of nineteenth-century

\footnotetext{
P. Haggard $(\bowtie)$

Institute of Cognitive Neuroscience, University College London, London, UK

e-mail: p.haggard@ucl.ac.uk

H. Lau

Department of Psychology, Columbia University, New York, USA
}

German psychophysics to study when people experience the sense of conscious intention that can accompany some voluntary actions. This triggered a lively tradition of experimental work investigating the relationship between brain activity and the conscious experience of volition. The neural correlates of conscious volition continue to be actively investigated, as several papers in this special issue show. Perhaps the most important legacy of this tradition has been the insight that volition may simply be a variety of conscious experience, along the same lines as visual awareness or emotional states. If conscious volition can be measured, it should be accessible to the same kinds of psychophysiological inquiry as those other states. The second key development was the identification of a specific brain mechanism in animals and humans that could form the basis of volition. Kornhuber and colleagues recorded a "readiness potential" preceding voluntary actions and emanating from the frontal cortex. A series of lesion studies in primates by Passingham and colleagues demonstrated the key role of the medial frontal cortex in internally generated action, as opposed to reactions to external stimuli. Single-unit recordings in the medial frontal cortex of primates by Tanji and colleagues identified neurons that appeared to code for entire sequences of actions stored in long-term memory, and played out as a chain of individual movements in the absence of any external cue. Taken together, these developments confirmed the roles of specific frontal and prefrontal regions in the flexible, strategic control of complex behaviour. Such findings contribute to our understanding of the link between thought and action that lies at the heart of volition. The combination of measurement and mechanism has made volition an experimental, scientific issue, rather than a purely philosophical one. 


\section{Decomposing volition}

This special issue shows a range of ways in which neuroscientists, experimental psychologists and others have risen to this experimental challenge. What overall picture of volition emerges? The scientific study of volition seems to be in an early, data-intensive stage, and it is difficult to extract general theoretical currents. Here, we make just a few general comments to highlight two approaches in the current literature that seem to us particularly productive. One important approach has decomposed volition into a distinct set of components. In our view, four key components of volition include reward, memory, generativity and inhibition. Reward-based decision-making suggests that behavioural choices are based on expectations of reward guided by previous reinforcement. The role of memory in voluntary action has been remarkably neglected, perhaps because it is difficult to study in an ecologically valid way. Action choices are often based on stored information about what one has just done or on what happened on previous occasions in similar contexts. Thus, in the absence of direct external instruction, memory of previous experience plays a crucial role in action decision. Generativity refers to the tendency to initiate and produce behaviour. This concept may be required to explain why actions occur in the first place. It is strongly linked to the continuum of neural excitability, from hypokinetic to hyperkinetic, that is postulated in neurology of movement disorders and in neuropsychiatry. The concept can be readily extended from motor generativity to cognitive generativity. Psychologists distinguish between exploratory, innovative behaviours, and exploitative, stereotyped behaviours. The long tradition of linking volition to the capacity for randomness builds on the capacity to generate innovative behaviour. A final and controversial component of volition is the capacity to inhibit action. Several accounts have sought to replace "free will" with "free won't". Some of these have involved an implausible dualism, in which a form of conscious causation, somehow independent of the brain, is thought to provide an ultimate veto over action. In fact, the last few decades of neuropsychological research have consistently demonstrated the importance of inhibitory control of behaviour by the frontal lobes. Inhibition is challenging to study, largely because it produces no behavioural output. However, the growing body of research focussing on inhibition of action is beginning to provide mechanistic insights into self-control.

\section{Volition as process: bottom-up or top-down views}

An alternative way to consider volition emphasises process, rather than components. We can distinguish two strongly divergent views of volitional processes in the literature.
According to a bottom-up view, voluntary actions are simply the results of fluctuating levels of neural activity in the motor system. This view rejects the concepts of causal triggers or decisions prior to voluntary action. Instead, actions occur when the random walk of neural activity crosses some point of no return or motor threshold. On this view, our voluntary actions happen to us, rather than us causing them. For example, Schurger et al. recently argued that the readiness potential is not a cause of voluntary action, but merely a trace of random neural activation that occasionally brings the motor system to the threshold for output. Bottom-up accounts of volition must explain why neural activation fluctuates. Neural noise intrinsic to the motor system is one possibility. Another would be a special circuit implementing the generativity component discussed above. Bottom-up accounts must either accept that voluntary action is not truly voluntary, but merely random, or must give an additional account to distinguish volition from randomness. One possibility is based on a form of "free won't". This view accepts the automatic generation of action impulses, but suggests that a form of top-down inhibitory control can stop unwanted impulses from triggering motor output. Alternatively, some currently unidentified mechanism might adjust the threshold level at which random neural fluctuations trigger motor output. On this view, volition would not primarily involve choosing what to do, but setting a criterion level for when and whether it would happen. Interestingly, recent work in metacognition suggests that criterion setting has a very general role in controlling conscious experience. We suggest that similar metacognitive processes could potentially contribute to the conscious experience of intention and control that accompanies some voluntary actions.

The top-down account of voluntary action processes is familiar to psychologists and neurologists interested in executive function. Classical cognitive models of controlled processes and supervisory attention can be readily recast as models of voluntary action. Most such models begin with a specific stimulus that functions as input to the model, whereas voluntary actions are "internally generated". In the case of voluntary action, the component of reward and motivation can be substituted for instruction or stimulus. Models of executive function are generally hierarchical and emphasise supervisory control. Further, they often avoid tackling issues of consciousness, even though executive processes are generally accompanied by conscious experience. For these two reasons, top-down models of volition processes risk being homuncular or even dualist. Clearly, volition may involve a mixture of both bottom-up and topdown processes. We hope that future research in this area will make increasing use of process models.

To summarise, volition is coming of age as a scientific topic. For these reasons, we have assembled a special 
issue, based on an open call for experimental papers, and a series of invited reviews by experts. Experimental Brain Research is the ideal venue for this snapshot of current research, since it has published many of the primary papers that provided the development of the field. We are grateful to all the authors who have contributed their work, particularly those who have worked on reviewing the volition literature. We also wish to thank the editorial and production teams who have made the process possible. 